Ciência e Natura, Santa Maria, v. 37 Ed. Especial PROFMAT, 2015, p. 79 - 88

Revista do Centro de Ciências Naturais e Exatas - UFSM

ISSN impressa: 0100-8307 ISSN on-line: 2179-460X

CIÊNCIAPNATURA 35䇾

\title{
Estudo dos Polígonos no plano de Argand-Gauss
}

Study of polygons in the Argand-Gauss plane

Felix Silva Costa e Robert Batista Pinheiro

\begin{abstract}
Resumo
Neste trabalho, apresentamos a parte histórica dos números complexos, mostrando o seu surgimento e sua evolução ao longo dos tempos. Classificamos os triângulos no plano de Argand-Gauss, levando em consideração os seus vértices e discutimos alguns resultados envolvendo o cálculo de área para polígonos convexos, em especial, uma fórmula para determinarmos a área de polígonos cujos vértices correspondem as raízes de um número complexo.
\end{abstract}

Palavras-chave: Números Complexos. Triângulos. Polígonos. Áreas

\begin{abstract}
In this work, we present the historical part of the complex numbers, showing its emergence and its evolution over time. We classify the triangles in the Argand-Gauss plane, considering its vertices and we discut some results involving the calculus of area to convex polygons, in particular, a formula to determine the area of polygons whose vertices correspond to the roots of a complex number.
\end{abstract}

Keywords: Complex number. Triangles. Polygons. Areas 


\section{Introdução}

Apesar do seu pouco aparecimento no ensino médio, os números complexos emergem naturalmente em diversas aplicações do mundo real. Eles possuem grande relevância em inúmeras áreas, principalmente nas engenharias, aparecem em grandezas como a impedância e a potência aparente, que são expressas por um número complexo. Já na aerodinâmica foi desenvolvida uma curva fechada no plano complexo que representa o perfil de uma asa de avião. Citamos também, uma aplicação na física, mais precisamente na óptica, onde determinamos a direção de um raio de luz refletido num espelho plano. ${ }^{1}$

Segundo Jucimar Peruzzo ${ }^{2}$, foi a partir do estudo das equações algébricas do $3^{\underline{o}}$ grau que se conseguiu um melhor entendimento da estrutura dos números complexos ou imaginários. Descobriu-se que o campo dos números reais é insuficiente para o estudo da álgebra, sendo indispensável trabalhar-se também com os números imaginários. Isso ficou bastante visível quanto à questão de resolução de equações. Os números complexos são uma das tantas abstrações matemáticas que facilitam o cálculo e a resolução de muitos problemas.

Neste artigo, fruto da dissertação Pinheiro (2012) de mestrado profissional em Matemática, em rede nacional, PROFMAT-MA, é discutido algumas propriedades dos polígonos convexos no plano de Argand-Gauss, apresentando uma expressão para calcular a área de um polígono convexo, em especial, obtemos uma fórmula para determinar a área de um polígono convexo regular, cujos vértices correspondem as raízes $n$-ésimas de um número complexo.

O trabalho está dividido da seguinte forma:

\footnotetext{
${ }^{1}$ Sistema óptico de espelhos constituído por superfícies planas e polidas, capazes de refletir regularmente a luz.

${ }^{2}$ Professor da E.E.B. Dom Felício César da Cunha Vasconcelos e E.E.B. Isabel da Silva Telles em, Irani, Santa Catarina.
}

a seção 2 apresenta um breve contexto hitórico sobre os números complexos, apresentando desde a sua origem até a evolução de sua representação; seção 3 apresenta a condição de alinhamento de três pontos no plano de Argand-Gauss; a seção 4 usa essa relação para classificar um triângulo formado nesse plano, a seção 5 calculamos a área de um triângulo nesse plano e finalmente a seção 6 apresenta uma fórmula para o cálculo da área de um polígono convexo, em especial, uma fórmula para calcular a área de um polígono regular em que os vértices são as raízes n-ésimas de um número complexo.

\section{Um pouco da história dos números complexos}

De acordo com Hellmeister (2004); Pinto (2009), uma das motivações principais do surgimento dos números complexos não foi à resolução de equações do $2^{\circ}$ grau cujas soluções são expressas por raízes quadradas de números negativos. O surgimento desses números está ligado diretamente à resolução de equações algébricas do $3^{\circ}$ grau. Até aquele momento, as equações do $2^{o}$ grau que apresentavam raízes quadradas de números negativos eram consideradas sem resolução e o objetivo dos matemáticos da época era desenvolver uma fórmula de resolução de equações cúbicas através de radicais, semelhante à que se usava para resolver as equações quadráticas.

Por volta de 1510, o matemático italiano Scipione Del Ferro (1465-1526) desenvolveu uma fórmula para um caso especial de cúbicas, ele desenvolveu uma resolução para equações do tipo

$$
x^{3}+p x+q=0
$$

Entretanto Scipione faleceu sem publicar seus resultados, divulgando-os apenas para um pequeno grupo de pessoas dentre eles Antônio Maria Del Fiore. De posse desse resultado Fiore tentou ganhar seu espaço entre os ma- 
temáticos e no ano de 1535 desafiou Nicolau Fontana (1500-1557), conhecido como Tartáglia, para um duelo de resolução de problemas (o que era comum naquela época). O desafio consistia na resolução de trinta problemas para cada, propostos pelo oponente, cabia ao perdedor pagar trinta banquetes. Tartáglia estava começando a se destacar no cenário matemático da época e prontamente aceitou o desafio. Todos os problemas propostos por Fior para Tartáglia recaiam no caso particular da cúbica cuja solução ele conhecia, Tartáglia sabia que seu adversário conhecia tal solução, mas o que Fior não sabia era que no dia 10 de fevereiro de 1535 Tartáglia também deduziu a fórmula e foi ainda mais longe deduzindo a fórmula para a equação do tipo

$$
x^{3}+p x^{2}+q=0
$$

Tartáglia venceu o desafio sem muito esforço, pois conseguiu resolver todos os problemas propostos por Fior, já seu adversário não conseguiu resolver nem a metade dos problemas impostos por Tartáglia, e saiu humilhado do desafio. A vitória de Tartáglia e sua descoberta ganham grande repercussão entre os matemáticos e ele recebe um convite de Girolamo Cardano (1501-1576) para ir a sua casa. Nessa época, Cardano gozava de boa posição em Milão e o convite era com o pretexto de apresentálo ao comandante militar da cidade, uma vez que Tartáglia tinha feito também grandes descobertas sobre tiros e fortificações, a intenção de Cardano era ganhar a autorização de Tartáglia para publicar a resolução da equação em seu livro Practica Arithmetica (1539). A princípio Tartáglia não revela a fórmula, mas depois de muita insistência Cardano conseguiu a fórmula com a promessa de que não publicaria, pois o próprio Tartáglia queria publicar e só estava esperando o momento exato para isso. Porém Tartáglia tinha revelado o segredo na forma de um poema cifrado e Cardano não conseguiu decifrar. Depois de mais promessas por parte de Cardano, Tartáglia revela a fórmula sem códigos, entretanto Cardano não cumpre a promessa e, em 1545, publica a fórmula de Tartáglia no seu livro Ars Magna.

A solução da equação cúbica do tipo $x^{3}+$ $p x+q=0$ se dava através da fórmula

$$
x=\sqrt[3]{-\frac{q}{2}+\sqrt{\left(\frac{q}{2}\right)^{2}+\left(\frac{p}{3}\right)^{3}}}+\sqrt[3]{-\frac{q}{2}-\sqrt{\left(\frac{q}{2}\right)^{2}+\left(\frac{p}{3}\right)^{3}}}
$$

conhecida hoje como fórmula de Cardano. Essa fórmula só se aplicava quando

$$
\left(\frac{q}{2}\right)^{2}+\left(\frac{p}{3}\right)^{3} \geq 0
$$

pois isso garantia a existência da raiz evitando assim a mesma situação das equações do $2^{\circ}$ grau com raízes quadradas de números negativos.

Nessa mesma época o matemático italiano Rafael Bombelli (1526-1573) em seu livro L'Álgebra Parte Maggiore Dell' Arithmetica fez um estudo sobre a resolução de equações de grau inferior a quatro e resolvendo a equação $x^{3}-$ $15 x=4$ verificou por inspeção que $x=4$ era solução da equação, pois $4^{3}-15.4=4$ mas, tentando verificar se encontrava a mesma solução $x=4$, pela fórmula de Cardano- Tartáglia obteve:

$$
\begin{aligned}
& x=\sqrt[3]{\frac{4}{2}+\sqrt{\frac{16}{4}-\frac{3375}{27}}}+\sqrt[3]{\frac{4}{2}-\sqrt{\frac{16}{4}-\frac{3375}{27}}}= \\
& =\sqrt[3]{2+\sqrt{-121}}+\sqrt[3]{2-\sqrt{-121}}
\end{aligned}
$$

Com esse resultado, Bombelli chegou a um impasse pois $\sqrt{-121}$ não existia. Logo, a equação não teria solução, mas $x=4$ era uma solução da equação pois satisfazia a igualdade. Somente em 1572 ele resolveu o impasse, partindo da hipótese que existiam expressões das formas $a+\sqrt{-b}$ e $a-\sqrt{-b}$ que seriam as formas simplificadas de, respectivamente, $\sqrt[3]{2+\sqrt{-121}}$ e $\sqrt[3]{2-\sqrt{-121}}$ tal que

$$
(a+\sqrt{-b})+(a-\sqrt{-b})=4
$$

concluindo assim que $a=2$. Faltava então determinar o valor de $b$, voltando a equação 
ele deduziu:

$$
\begin{aligned}
& 2+\sqrt{-b}=\sqrt[3]{2+\sqrt{-121}}=\sqrt[3]{2+\sqrt{121} \cdot \sqrt{-1}}= \\
& \sqrt[3]{2+11 \cdot \sqrt{-1}}
\end{aligned}
$$

Elevando ambos os membros ao cubo, chegou na seguinte relação:

$$
(8-6 b)+(12-b) \sqrt{-b}=2+11 \sqrt{-1} .
$$

Por comparação concluiu que $b=1$. Assim:

$$
\begin{aligned}
& \sqrt[3]{2+\sqrt{-121}}=2+\sqrt{-1} \mathrm{e} \\
& \sqrt[3]{2-\sqrt{-121}}=2-\sqrt{-1}
\end{aligned}
$$

Portanto

$$
\begin{aligned}
& x=\sqrt[3]{2+\sqrt{-121}}+\sqrt[3]{2-\sqrt{-121}} \\
& =2+\sqrt{-1}+2-\sqrt{-1}=4 .
\end{aligned}
$$

Bambelli também criou as regras da multiplicação para operar com $\sqrt{-1}$, ou seja:

$$
\begin{gathered}
(\sqrt{-1}) \cdot(\sqrt{-1})=-1 \\
(-\sqrt{-1}) \cdot(\sqrt{-1})=1 \\
(-\sqrt{-1}) \cdot(-\sqrt{-1})=-1 \\
( \pm 1)(\sqrt{-1})= \pm \sqrt{-1} \\
( \pm 1)( \pm \sqrt{-1})= \pm \sqrt{-1}
\end{gathered}
$$

Além dessa regra da multiplicação também criou a regra para somar dois números da forma $x+y \sqrt{-1}, x, y \in \mathbb{R}$, ou seja:

$$
\begin{aligned}
& \left(x_{1}+y_{1} \sqrt{-1}\right)+\left(x_{2}+y_{2} \sqrt{-1}\right)= \\
& =\left(x_{1}+x_{2}\right)+\left(y_{1}+y_{2}\right) \sqrt{-1} .
\end{aligned}
$$

É importante ressaltar que a notação $\sqrt{-1}$ só foi introduzida no ano de 1629 por Albert Girard (1595-1632) em seu livro L'Invention Nouvelle em Algebre, até então a simbologia para $\sqrt{-1}$ era da forma $R[0 \mathrm{~m} .1]$ ( $R$ de raiz, $m$ de menos) usada por Bombelli. Já os termos real e imaginário foram introduzidos em 1637 por René Descartes (1596-1650); em 1777 Leonhard Euler (1707-1783) introduziu o símbolo $i$ para denotar $\sqrt{-1}$ sendo aceito plenamente somente no ano de 1801 quando Friederich Gauss (1777-1855) começou a usar de forma contínua essa notação. Também foi Gauss que introduziu a expressão "números complexos" e coube a Willian Rowan Hamilton (1805-1865) a introduzir a notação $a+b i$ para um número complexo, essa expressão é denominada nos dias de hoje como forma algébrica de um número complexo.

No campo geométrico o desenvolvimento aconteceu a partir do ano de 1800 quando Jean Robert Argand (1768-1822) e Gauss chegaram a conclusão que os números complexos poderiam ser representados em um sistema de coordenadas retangulares e para isso eles convencionaram que o eixo horizontal representaria os números reais, o eixo vertical representaria os números imaginários e qualquer complexo poderia ser representado na forma de par ordenado, isto é, $a+b i$ corresponderia ao par $(a, b)$. Com essas conversões um complexo $a+b i$ representaria geometricamente um ponto nesse plano bidimensional que hoje é denominado de plano de Argand-Gauss.

\section{Condição de alinhamento de três complexos}

vaaos os compıexos $z_{1}, z_{2}$ e $z_{3}$ tais que $J\left(z_{1}\right)=$ $P, f\left(z_{2}\right)=Q$ e $f\left(z_{3}\right)=R, \operatorname{com} P, Q, R \in \mathbb{R}^{2}$, a condição para que esses três pontos estejam alinhados Viggiani (1968) é que exista $\lambda \in \mathbb{R}$, tal que $z_{3}-z_{1}=\lambda\left(z_{2}-z_{1}\right)$. Sendo $z_{2} \neq z_{1}$,

$$
\lambda=\frac{z_{3}-z_{1}}{z_{2}-z_{1}} .
$$

Lembrando que se $z$ é real, então $z=\bar{z}$, obtemos:

$$
\frac{z_{3}-z_{1}}{z_{2}-z_{1}}=\overline{\left(\frac{z_{3}-z_{1}}{z_{2}-z_{1}}\right)}=\frac{\overline{z_{3}}-\overline{z_{1}}}{\overline{z_{2}}-\overline{z_{1}}} .
$$


Daí, segue que:

$$
\left(z_{3}-z_{1}\right) \cdot\left(\overline{z_{2}}-\overline{z_{1}}\right)-\left(z_{2}-z_{1}\right) \cdot\left(\overline{z_{3}}-\overline{z_{1}}\right)=0 .
$$

Desenvolvendo,

$$
\begin{aligned}
& z_{2} \overline{z_{3}}+z_{1} \overline{z_{2}}+z_{3} \overline{z_{1}}-\left(z_{1} \overline{z_{3}}+z_{3} \overline{z_{2}}+z_{2} \overline{z_{1}}\right)=0 \\
& \Longleftrightarrow\left|\begin{array}{ccc}
1 & 1 & 1 \\
z_{1} & z_{2} & z_{3} \\
\overline{z_{1}} & \overline{z_{2}} & \overline{z_{3}}
\end{array}\right|=0 .
\end{aligned}
$$

Logo,

$$
\left|\begin{array}{ccc}
1 & 1 & 1 \\
z_{1} & z_{2} & z_{3} \\
\overline{z_{1}} & \overline{z_{2}} & \overline{z_{3}}
\end{array}\right|=0
$$

É a condição necessária e suficiente para que $z_{1}, z_{2}$ e $z_{3}$ sejam colineares.

\section{Classificação dos triângulos}

Na relação $\lambda=\frac{z_{3}-z_{1}}{z_{2}-z_{1}}$, se $\lambda \in \mathbb{R}$ então $z_{1}, z_{2}$ e $z_{3}$ são colineares, caso contrário os seus afixos são vértices de um triângulo Viggiani (1968).

Seja o complexo $d=\frac{z_{3}-z_{1}}{z_{2}-z_{1}}$ denominado de relação simples de três complexos, onde $|d|=$ $\frac{\left|z_{3}-z_{1}\right|}{\left|z_{2}-z_{1}\right|}$ e $\arg (d)=\theta$, onde o ângulo $\theta$ é formado pelos lados $\overline{z_{1} z_{2}}$ e $\overline{z_{1} z_{3}}{ }^{3}$.

Observamos que $\arg (d)=\arg \left(\frac{z_{3}-z_{1}}{z_{2}-z_{1}}\right)=$ $\arg \left(z_{3}-z_{1}\right)-\arg \left(z_{2}-z_{1}\right)$. Sendo $\arg \left(z_{3}-z_{1}\right)$, $\arg \left(z_{2}-z_{1}\right)$ os ângulos dos vetores $z_{3}-z_{1}$, $z_{2}-z_{1}$, então $\arg \left(z_{3}-z_{1}\right)=\arg \left(z_{2}-z_{1}\right)+\theta$.

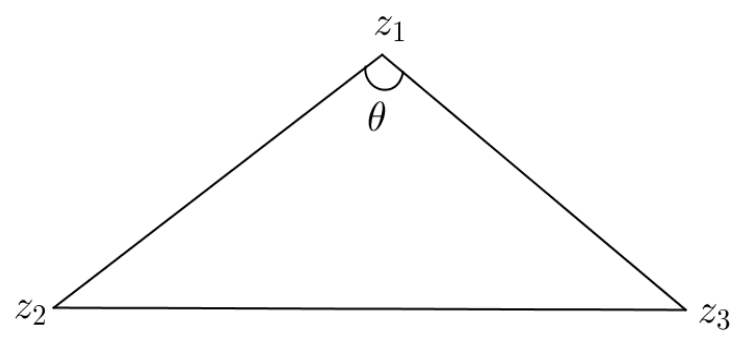

Figura 1: Triângulo 1

\footnotetext{
${ }^{3}$ Se optássemos por trabalhar com o ângulo formado pelos lados $\overline{z_{2} z_{3}}$ e $\overline{z_{2} z_{1}}$ a relação seria $d=\frac{z_{3}-z_{2}}{z_{1}-z_{2}}$.
}

Analisando o triângulo da Figura 1 conforme a relação $d=\frac{z_{3}-z_{1}}{z_{2}-z_{1}}$ :

1. Se $|d|=1 \Longrightarrow\left|z_{3}-z_{1}\right|=\left|z_{2}-z_{1}\right| \Longrightarrow$ $\Delta z_{1} z_{2} z_{3}$ é isósceles;

2. Se $d$ for imaginário puro, então $\cos \theta=0$. De fato, $\operatorname{como} \cos \theta=\frac{a}{|d|}$ e $d$ é imaginário puro, então $a=0$, uma vez que $a$ é a parte real de $d$. Dessa forma, $\theta=\frac{\pi}{2} \mathrm{rad}$ e, consequentemente, $\Delta z_{1} z_{2} z_{3}$ é retângulo em $z_{1}$;

3. Se $d$ for real, temos a condição de alinhamento e, em consequência, os afixos $z_{1}, z_{2}$ e $z_{3}$ não formam um triângulo.

4. Se $d=\frac{1}{2}+\frac{\sqrt{3}}{2} i$, temos que $|d|=\sqrt{\frac{1}{4}+\frac{3}{4}}=$ 1 , consequentemente

$$
\left|z_{3}-z_{1}\right|=\left|z_{2}-z_{1}\right| \text { e } \theta=\frac{\pi}{3} \mathrm{rad} .
$$

Dessa forma, $\Delta z_{1} z_{2} z_{3}$ é equilátero.

Portanto, classificamos um triângulo no plano de Argand-Gass, de acordo com a variável $d$. Aqui, a referência utilizada neste capítulo.

\section{5 Área do triângulo}

Após classificar os triângulos, vamos determinar a área de um triângulo qualquer, levando em consideração os seus vértices.

Seja o triângulo $z_{1} z_{2} z_{3}$ e $N_{r}, r=1,2,3$, a projeção de qualquer ponto de $z_{r}$ sobre o eixo $O x$. Dessa forma, temos:

$$
\begin{aligned}
& A\left(\Delta P_{1} P_{2} P_{3}\right)= \\
& =A\left(P_{1} P_{2} N_{1} N_{2}\right)+A\left(P_{1} P_{3} N_{3} N_{1}\right)-A\left(P_{2} N_{2} N_{3} P_{3}\right) \\
& =\frac{1}{2}\left(P_{2} N_{2}+P_{1} N_{1}\right) N_{2} N_{1}+ \\
& +\frac{1}{2}\left(P_{1} N_{1}+P_{3} N_{3}\right) N_{1} N_{3}-\frac{1}{2}\left(P_{2} N_{2}+P_{3} N_{3}\right) N_{2} N_{3} \\
& =\frac{1}{2}\left(y_{2}+y_{1}\right)\left(x_{1}-x_{2}\right)+\frac{1}{2}\left(y_{1}+y_{3}\right)\left(x_{3}-x_{1}\right)+ \\
& -\frac{1}{2}\left(y_{2}+y_{3}\right)\left(x_{3}-x_{2}\right) \\
& =\frac{1}{2}\left[\left(x_{1} y_{2}-y_{1} x_{2}\right)+\left(x_{2} y_{3}-y_{2} x_{3}\right)+\left(x_{3} y_{1}-y_{3} x_{1}\right)\right],
\end{aligned}
$$




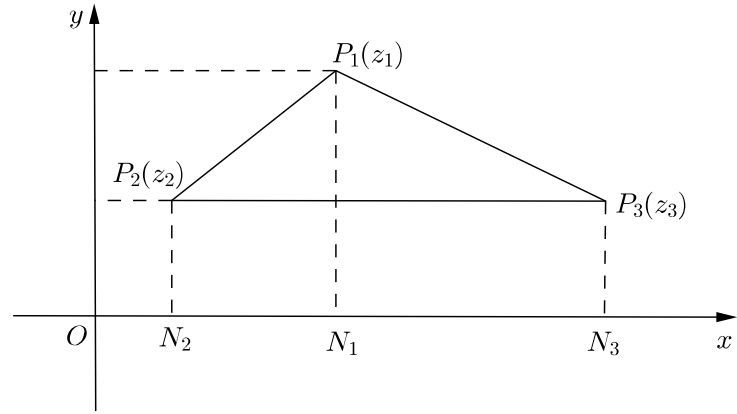

Figura 2: Triângulo 2.

onde $P_{1} P_{2} N_{1} N_{2}, P_{1} P_{3} N_{3} N_{1}, P_{2} N_{2} N_{3} P_{3}$ representam trapézios. Observando que,

$$
\begin{aligned}
& \text { i) } x_{1} y_{2}-y_{1} x_{2}= \\
& =\operatorname{Im}\left[\left(x_{1}-i y_{1}\right)\left(x_{2}+i y_{2}\right)\right] \\
& =\operatorname{Im}\left(\overline{z_{1}} z_{2}\right)=\frac{1}{2} i\left(z_{1} \overline{z_{2}}-\overline{z_{1}} z_{2}\right) \\
& \text { ii) } x_{2} y_{3}-y_{2} x_{3}= \\
& =\operatorname{Im}\left[\left(x_{2}-i y_{2}\right)\left(x_{3}+i y_{3}\right)\right] \\
& =\operatorname{Im}\left(\overline{z_{2}} z_{3}\right)=\frac{1}{2} i\left(z_{2} \overline{z_{3}}-\overline{z_{2}} z_{3}\right) \\
& \text { iii) } x_{3} y_{1}-y_{3} x_{1}= \\
& =\operatorname{Im}\left[\left(x_{3}-i y_{3}\right)\left(x_{1}+i y_{1}\right)\right] \\
& =\operatorname{Im}\left(\overline{z_{3}} z_{1}\right)=\frac{1}{2} i\left(z_{3} \overline{z_{1}}-\overline{z_{3}} z_{1}\right),
\end{aligned}
$$

então:

$$
\begin{aligned}
& A\left(\Delta P_{1} P_{2} P_{3}\right) \\
& =\frac{i}{4}\left(z_{1} \overline{z_{2}}-\overline{z_{1}} z_{2}+z_{2} \overline{z_{3}}-\overline{z_{2}} z_{3}+z_{3} \overline{z_{1}}-\overline{z_{3}} z_{1}\right) \\
& =\frac{i}{4}\left|\begin{array}{ccc}
1 & 1 & 1 \\
z_{1} & z_{2} & z_{3} \\
\overline{z_{1}} & \overline{z_{2}} & \overline{z_{3}}
\end{array}\right| .
\end{aligned}
$$

Este determinante é puramente imaginário, já que

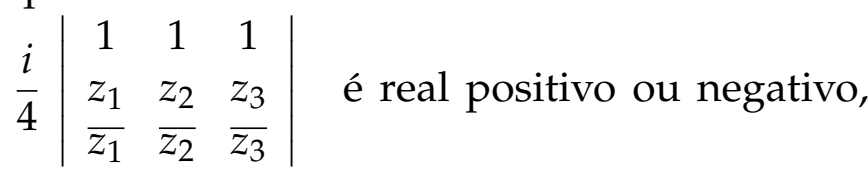
caso seja negativo devemos tomar seu módulo.

$\mathrm{O}$ determinante acima pode ser reescrito como

$$
\left|\begin{array}{ll}
z_{1} & z_{2} \\
\overline{z_{1}} & \overline{z_{2}}
\end{array}\right|+\left|\begin{array}{ll}
z_{2} & z_{3} \\
\overline{z_{2}} & \overline{z_{3}}
\end{array}\right|+\left|\begin{array}{ll}
z_{3} & z_{1} \\
\overline{z_{3}} & \overline{z_{1}}
\end{array}\right| \text {, para isso }
$$

basta aplicar o teorema de Laplace na primeira linha, ou seja:

$$
\begin{aligned}
& \left|\begin{array}{ccc}
1 & 1 & 1 \\
z_{1} & z_{2} & z_{3} \\
\overline{z_{1}} & \overline{z_{2}} & \overline{z_{3}}
\end{array}\right|=a_{11} \cdot A_{11}+a_{12} \cdot A_{12}+a_{13} \cdot A_{13}= \\
& =\left|\begin{array}{cc}
z_{2} & z_{3} \\
\overline{z_{2}} & \overline{z_{3}}
\end{array}\right|-\left|\begin{array}{cc}
z_{1} & z_{3} \\
\overline{z_{1}} & \overline{z_{3}}
\end{array}\right|+\left|\begin{array}{cc}
z_{1} & z_{2} \\
\overline{z_{1}} & \overline{z_{2}}
\end{array}\right|,
\end{aligned}
$$

onde $A_{i j}$ é o cofator do elemento $a_{i j}, i, j=1,2,3$, da matriz $\left(\begin{array}{ccc}1 & 1 & 1 \\ z_{1} & z_{2} & z_{3} \\ \frac{z_{1}}{z_{2}} & \frac{\bar{z}}{z_{3}}\end{array}\right)$.
Como $\left|\frac{z_{1}}{z_{1}} \frac{z_{3}}{z_{3}}\right|=-\left|\begin{array}{ll}\frac{z_{3}}{z_{3}} & \frac{z_{1}}{z_{1}}\end{array}\right|$, então:

$$
\begin{aligned}
& \left|\begin{array}{ccc}
1 & 1 & 1 \\
z_{1} & z_{2} & z_{3} \\
\overline{z_{1}} & \overline{z_{2}} & \overline{z_{3}}
\end{array}\right|= \\
& =\left|\begin{array}{ll}
z_{1} & z_{2} \\
\overline{z_{1}} & \overline{z_{2}}
\end{array}\right|+\left|\begin{array}{ll}
z_{2} & z_{3} \\
\overline{z_{2}} & \overline{z_{3}}
\end{array}\right|+\left|\begin{array}{cc}
z_{3} & z_{1} \\
\overline{z_{3}} & \overline{z_{1}}
\end{array}\right| .
\end{aligned}
$$

Logo, a área do triângulo $\Delta P_{1} P_{2} P_{3}$ também pode ser dada pela expressão:

$$
\begin{aligned}
& A\left(\Delta P_{1} P_{2} P_{3}\right)= \\
& =\frac{i}{4}\left(\left|\begin{array}{ll}
z_{1} & z_{2} \\
\overline{z_{1}} & \overline{z_{2}}
\end{array}\right|+\left|\begin{array}{ll}
z_{2} & z_{3} \\
\overline{z_{2}} & \overline{z_{3}}
\end{array}\right|+\left|\begin{array}{ll}
z_{3} & z_{1} \\
\overline{z_{3}} & \overline{z_{1}}
\end{array}\right|\right) .
\end{aligned}
$$

\section{6 Área de um polígono convexo}

A partir de (2), vamos determinar uma fórmula para o cálculo da área de um polígono convexo formado por $n$ pontos, baseado em Costa (2008), que apresenta uma demonstração detalhada no caso dos vértices do polígono pertencerem ao plano $\mathbb{R}^{2}$. Entretanto, devemos lembrar que a área de um polígono convexo é igual à soma das áreas de uma decomposição do polígono em triângulos. Consideramos um polígono convexo $P$ de $n$ lados e de pontos $P_{1}, P_{2}, \ldots, P_{n}$, percorridos no sentido anti-horário, $A_{\text {polígono }}=A_{P_{1} P_{2} P_{3}}+A_{P_{1} P_{3} P_{4}}+\ldots+A_{P_{1} P_{n-1} P_{n}}$ onde $A_{P_{1} P_{k} P_{k+1}}$ é a área do $k$-ésimo triângulo, 


$$
\begin{aligned}
& A_{P_{1} P_{k} P_{k+1}}= \\
& =\frac{i}{4}\left(\left|\begin{array}{ll}
z_{1} & z_{k} \\
\overline{z_{1}} & \overline{z_{k}}
\end{array}\right|+\left|\begin{array}{ll}
z_{k} & z_{k+1} \\
\overline{z_{k}} & \overline{z_{k+1}}
\end{array}\right|+\left|\begin{array}{ll}
z_{k+1} & z_{1} \\
\overline{z_{k+1}} & \overline{z_{1}}
\end{array}\right|\right) .
\end{aligned}
$$

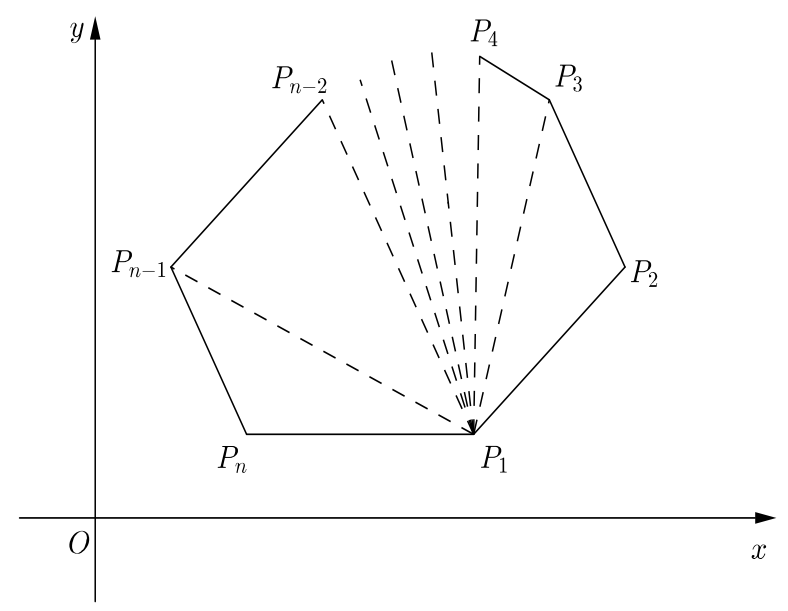

Figura 3: Polígono convexo no plano de Argand-Gauss.

Então da equação (3), temos:

$$
\begin{aligned}
& A_{\text {polígono }}= \\
& =\frac{i}{4}\left(\left|\begin{array}{ll}
z_{1} & z_{2} \\
\overline{z_{1}} & \overline{z_{2}}
\end{array}\right|+\left|\begin{array}{cc}
z_{2} & z_{3} \\
\overline{z_{2}} & \overline{z_{3}}
\end{array}\right|+\left|\begin{array}{cc}
z_{3} & z_{1} \\
\overline{z_{3}} & \overline{z_{1}}
\end{array}\right|\right)+ \\
& +\frac{i}{4}\left(\left|\begin{array}{cc}
z_{1} & z_{3} \\
\overline{z_{1}} & \overline{z_{3}}
\end{array}\right|+\left|\begin{array}{cc}
z_{3} & z_{4} \\
\overline{z_{3}} & \overline{z_{4}}
\end{array}\right|+\left|\begin{array}{cc}
z_{4} & z_{1} \\
\overline{z_{4}} & \overline{z_{1}}
\end{array}\right|\right)+ \\
& +\frac{i}{4}\left(\left|\begin{array}{ll}
z_{1} & z_{4} \\
\overline{z_{1}} & \overline{z_{4}}
\end{array}\right|+\left|\begin{array}{cc}
z_{4} & z_{5} \\
\overline{z_{4}} & \overline{z_{5}}
\end{array}\right|+\left|\begin{array}{cc}
z_{5} & z_{1} \\
\overline{z_{5}} & \overline{z_{1}}
\end{array}\right|\right)+\ldots \\
& +\frac{i}{4}\left(\left|\begin{array}{cc}
z_{1} & z_{n-1} \\
\overline{z_{1}} & \overline{z_{n-1}}
\end{array}\right|+\left|\begin{array}{lll}
z_{n-1} & z_{n} \\
\overline{z_{n-1}} & \overline{z_{n}}
\end{array}\right|+\left|\begin{array}{cc}
\frac{z_{n}}{z_{n}} & z_{1} \\
z_{1}
\end{array}\right|\right) .
\end{aligned}
$$

Observamos na equação (4) que o último determinante de $A_{P_{1} P_{k} P_{k+1}}$ se anula com o primeiro determinante de $A_{P_{1} P_{k+1} P_{k+2}}$. Logo,

$$
\begin{aligned}
& A_{\text {polígono }}= \\
& =\frac{i}{4}\left(\left|\frac{z_{1}}{z_{1}} \frac{z_{2}}{z_{2}}\right|+\left|\begin{array}{ll}
z_{2} & z_{3} \\
z_{2} & \frac{z_{3}}{z_{3}}
\end{array}\right|+\ldots+\left|\begin{array}{cc}
z_{n} & z_{1} \\
z_{n} & \overline{z_{1}}
\end{array}\right|\right)
\end{aligned}
$$

\subsection{Caso particular}

Já sabemos que as raízes n-ésimas de $z \neq 0$ representam geometricamente os vértices de um polígono regular de $n$ lados. Representando essas raízes por $w_{0}, w_{2}, \ldots, w_{n-1}$, a área desse polígono formado pelos afixos dessas raízes é dada pela expressão.

$A_{\text {polígono }}=$
$=\frac{i}{4}\left(\left|\begin{array}{cc}\frac{w_{0}}{w_{0}} & \frac{w_{1}}{w_{1}}\end{array}\right|+\left|\begin{array}{cc}\frac{w_{1}}{w_{1}} & \frac{w_{2}}{w_{2}}\end{array}\right|+\ldots+\left|\begin{array}{ll}\frac{w_{n-1}}{w_{n-1}} & \frac{w_{0}}{w_{0}}\end{array}\right|\right)$.

Vimos que (5) determina a área do polígono regular formado pelas raízes $n$-ésimas de um complexo $a+b i$, então será que seria possível chegar a uma equação que determina essa mesma área sem ter que calcular essas raízes? Isto é, seria possível determinar uma fórmula que calcula essa área que dependa apenas de $a, b$ e $n$ ? Vejamos:

Sabemos que a raiz $n$-ésima de um número complexo z é dada por Capelas de Oliveira (2006):

$$
w_{k}=\rho\left[\cos \left(\frac{\theta+2 k \pi}{n}\right)+i \operatorname{sen}\left(\frac{\theta+2 k \pi}{n}\right)\right]
$$

onde, $k=0,1, \ldots, n-1, \rho=\sqrt[n]{|z|}=\sqrt[2 n]{a^{2}+b^{2}}, \theta=$ $\operatorname{arctg}\left(\frac{b}{a}\right)$. Usando a equação (6), determinamos o k-ésimo determinante da equação (5),

$$
\begin{aligned}
& \left|\begin{array}{ll}
\frac{w_{k-1}}{w_{k-1}} & \frac{w_{k}}{w_{k}}
\end{array}\right|= \\
& =\rho^{2}\left|\begin{array}{cc}
1 & i \\
1 & -i
\end{array}\right|\left|\begin{array}{cc}
\cos \left(\frac{\theta+2(k-1) \pi}{n}\right) & \cos \left(\frac{\theta+2 k \pi}{n}\right) \\
\operatorname{sen}\left(\frac{\theta+2(k-1) \pi}{n}\right) & \operatorname{sen}\left(\frac{\theta+2 k \pi}{n}\right)
\end{array}\right| \\
& =-2 i \rho^{2}\left[\operatorname{sen}\left(\frac{\theta+2 k \pi}{n}\right) \cos \left(\frac{\theta+2(k-1) \pi}{n}\right)+\right. \\
& \left.-\operatorname{sen}\left(\frac{\theta+2(k-1) \pi}{n}\right) \cos \left(\frac{\theta+2 k \pi}{n}\right)\right] \\
& =-2 i \rho^{2} \operatorname{sen}\left(\frac{2 \pi}{n}\right) .
\end{aligned}
$$

Portanto, como o resultado obtido na equação (7) independe do $k$, os demais determinantes da equação (6) possuem o mesmo resultado. Além disso, como temos $n$ determinantes, então multiplicamos o valor da equação (7) por 
n, para obtermos a área do polígono. Ou seja, a área do polígono é:

$$
\begin{aligned}
A_{\text {polígono }} & =n \frac{i}{4}\left[-2 i \rho^{2} \operatorname{sen}\left(\frac{2 \pi}{n}\right)\right] \\
& =\frac{n \rho^{2}}{2} \operatorname{sen}\left(\frac{2 \pi}{n}\right) \\
& =\frac{n \sqrt[n]{a^{2}+b^{2}} \operatorname{sen}\left(\frac{2 \pi}{n}\right)}{2}
\end{aligned}
$$

Outra forma de calcularmos esta expressão é: seja $w_{0}, w_{1}, w_{2} \cdots w_{n-1}$ as raízes $n$-ésimas de $z=a+b i$ e seja $l$ a medida do lado do polígono regular formado por essas raízes, então:

$$
\begin{aligned}
& \left|w_{1}-w_{0}\right|=\left|w_{2}-w_{1}\right|=\cdots \\
& =\left|w_{k}-w_{k-1}\right|=\cdots=\left|w_{n-1}-w_{0}\right|=l .
\end{aligned}
$$

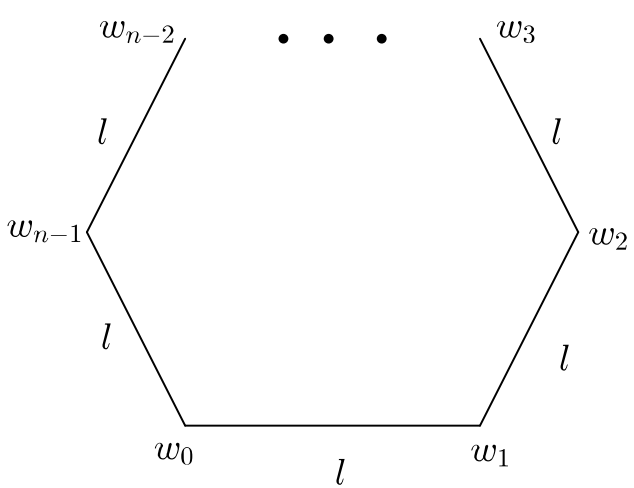

Figura 4: Polígono regular formado pelas raízes n-ésimas.

Logo, $l=\left|w_{k}-w_{k-1}\right|$. Como o valor de $w_{k}$ é dado por

$$
w_{k}=\rho\left[\cos \left(\frac{\theta+2 k \pi}{n}\right)+i \operatorname{sen}\left(\frac{\theta+2 k \pi}{n}\right)\right]
$$

e o valor de $w_{k-1}$ é dado por

$$
w_{k-1}=
$$$$
=\rho\left[\cos \left(\frac{\theta+2 k \pi-2 \pi}{n}\right)+i \operatorname{sen}\left(\frac{\theta+2 k \pi-2 p i}{n}\right)\right],
$$
onde $\rho=\sqrt[n]{|z|}=\sqrt[2 n]{a^{2}+b^{2}}, \theta=\operatorname{arctg}\left(\frac{b}{a}\right) \mathrm{e}$ $k \in \mathbb{Z}$, fazendo $w_{k}-w_{k-1}$, obtemos:

$$
w_{k}-w_{k-1}=
$$

$=\rho\left[\cos \left(\frac{\theta+2 k \pi}{n}\right)-\cos \left(\frac{\theta+2 k \pi-2 \pi}{n}\right)\right]+$

$+i \rho\left[\operatorname{sen}\left(\frac{\theta+2 k \pi}{n}\right)-\operatorname{sen}\left(\frac{\theta+2 k \pi-2 p i}{n}\right)\right]$

$=\rho\left[-2 \operatorname{sen}\left(\frac{2 \theta+4 k \pi-2 \pi}{2 n}\right) \operatorname{sen}\left(\frac{\pi}{n}\right)+\right.$

$\left.+2 i \operatorname{sen}\left(\frac{\pi}{n}\right) \cos \left(\frac{2 \theta+4 k \pi-2 \pi}{2 n}\right)\right]$.

$=2 \rho \operatorname{sen}\left(\frac{\pi}{n}\right)\left[-\operatorname{sen}\left(\frac{2 \theta+4 k \pi-2 \pi}{2 n}\right)+\right.$

$\left.+i \cos \left(\frac{2 \theta+4 k \pi-2 \pi}{2 n}\right)\right]$.

Aplicando o módulo,

$$
\begin{aligned}
& \left|w_{k}-w_{k-1}\right|= \\
= & 2 \rho \operatorname{sen}\left(\frac{\pi}{n}\right) \sqrt{\operatorname{sen}^{2}\left(\frac{2 \theta+4 k \pi-2 \pi}{2 n}\right)+\cos ^{2}\left(\frac{2 \theta+4 k \pi-2 \pi}{2 n}\right)} \\
= & 2 \rho \operatorname{sen}\left(\frac{\pi}{n}\right) .
\end{aligned}
$$

Logo, o lado do polígono é

$$
l=2 \rho \operatorname{sen}\left(\frac{\pi}{n}\right)=2 \sqrt[2 n]{a^{2}+b^{2}} \operatorname{sen}\left(\frac{\pi}{n}\right) .
$$

O polígono pode ser dividido em $n$ triângulos com centro numa circunferência circunscrita como um vértice comum a todos esses $n$ triângulos.

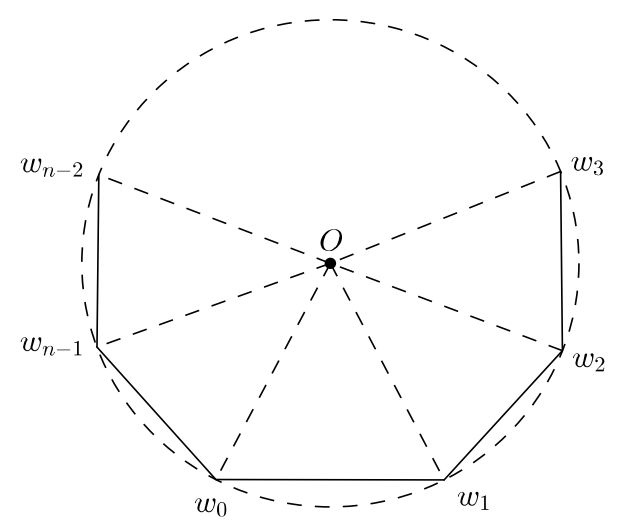

Figura 5: Polígono associado às raízes n-ésimas de um complexo, inscrito numa circunferência

Definindo $\rho$ como a medida do raio dessa circunferência circunscrita, então vamos calcular a área de um desses triângulos. 


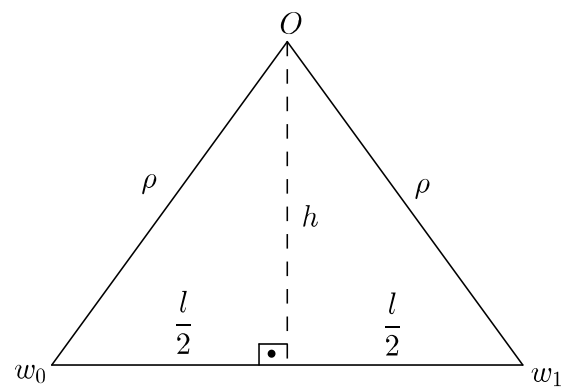

Figura 6: Triângulo 3.

Portanto,

$$
\begin{aligned}
& \rho^{2}=h^{2}+\left(\frac{l}{2}\right)^{2} \\
& \Longrightarrow h=\sqrt{\rho^{2}-\frac{l^{2}}{4}}=\sqrt{\rho^{2}-\frac{4 \rho^{2} \operatorname{sen}\left(\frac{\pi}{n}\right)}{4}}= \\
& =\sqrt{\rho^{2}-\rho^{2} \operatorname{sen}^{2}\left(\frac{\pi}{n}\right)} \\
& =\rho \sqrt{1-\operatorname{sen}^{2}\left(\frac{\pi}{n}\right)}=\rho \sqrt{\cos ^{2}\left(\frac{\pi}{n}\right)}=\rho \cos \left(\frac{\pi}{n}\right) .
\end{aligned}
$$

Logo, a área do triângulo será:

$$
A_{T}=\frac{h l}{2}=\frac{\rho \cos \left(\frac{\pi}{n}\right) 2 \rho \operatorname{sen}\left(\frac{\pi}{n}\right)}{2} .
$$

Como o polígono foi dividido em $n$ triângulos iguais, então:

$$
\begin{aligned}
& A_{\text {polígono }}=n A_{T}=n \frac{\rho^{2}\left[2 \cos \left(\frac{\pi}{n}\right) \operatorname{sen}\left(\frac{\pi}{n}\right)\right]}{2} \\
& =n \frac{\rho^{2}\left[\operatorname{sen}\left(\frac{2 \pi}{n}\right)\right]}{2} .
\end{aligned}
$$

Segue que, a área do polígono regular será:

$$
A_{\text {polígono }}=\frac{n \sqrt[n]{a^{2}+b^{2}} \operatorname{sen}\left(\frac{2 \pi}{n}\right)}{2} .
$$

Se reescrevermos a equação (8), da forma:

$$
A_{\text {polígono }}=\pi \frac{\operatorname{sen}\left(\frac{2 \pi}{n}\right)}{\frac{2 \pi}{n}} \sqrt[n]{a^{2}+b^{2}}
$$

Observe ainda que usando o conceito de limite na equação (9), que a área do polígono vale $\pi$, quando $n$ tende infinito. Basta usarmos a propriedade do limite fundamental trigonométrico e o limite da raiz n-ésima de um número, onde ambos valem 1 , com $n \rightarrow \infty$. Portanto, concluímos que a medida que $n$ cresce o polígono formado pelos vértices das raízes $n$-ésimas do número complexo, se aproxima de um círculo unitário, com centro na origem.

\section{Conclusões}

A forma como os números complexos surgem na matemática e sua gama de aplicações, sejam elas físicas ou de qualquer outra natureza, mostra o quanto eles são imprescindíveis e ao mesmo tempo fascinantes. Além de mostrarmos seu aparecimento e evolução, estabelecemos uma importante conexão entre os números complexos com outros tópicos do ensino médio, tais como trigonometria e geometria plana. Discutimos alguns resultados para polígonos convexos, em particular, os regulares formados a partir das raízes de um número complexo, no plano Argand-Gauss, mais propriamente, uma relação para o cálculo de área de polígonos convexos através dos números complexos. Destacamos ainda, que este trabalho é resultado da dissertação de metrado do programa de Mestrado Profissional em Matemática, PROFMATMA, como dito em seção anterior, que tem como objetivo primordial, mostrar a diversidade dos números complexos para os alunos e professores do ensino médio, ou seja, mostrar que os números complexos não estão isolados de outros conteúdos da matemática.

\section{Agradecimentos}

Agradecemos ao Dr. J. A. P. Ferreira Marão pelas diversas discussões e sugestões. 


\section{Referências}

Capelas de Oliveira, E. e Rodrigues Jr., W. A., 2006. Funções analíticas com aplicações. Livraria da Fśica.

Costa, F. S., 2008. Áreas e contornos. Dissertação de Mestrado, IMECC-UNICAMP.

Hellmeister, A. C. P. e Peixoto, C. M., 2004. Matemática: Ensino Médio. Ministério da Educação, Secretaria de Educação Básica.

Pinheiro, R. B., 2012. Números complexos: alguns aspectos algébricos e geométricos. Dissertação de Mestrado, UFMA.

Pinto, U. J., 2009. A história dos números complexos: das quantidades. Dissertação de Mestrado, UFRJ.

Viggiani, D., 1968. Alguns aspectos da aplicação dos números complexos a geometria. Tese de Doutorado, UNIMAR. 